PROCEEDINGS OF THE

AMERICAN MATHEMATICAL SOCIETY

Volume 126, Number 10, October 1998, Pages 2999-3005

S $0002-9939(98) 04658-9$

\title{
A NOTE ON THE GELFAND-MAZUR THEOREM
}

\author{
S. J. BHATT, D. J. KARIA, S. H. KULKARNI, AND M. E. SHIMPI
}

(Communicated by Palle E. T. Jorgensen)

\begin{abstract}
Three Gelfand-Mazur type theorems are proved. One of these provides a $C^{*}$-property analogue of Zalar's recent generalizations of the FroelichIngelstam-Smiley Theorems concerning unital multiplication in Hilbert spaces; the second illustrates that the assumption in Kaplansky's version of the Gelfand-Mazur Theorem can be weakened in the presence of a $C^{*}$-norm; whereas the third provides a real analogue of a result due to Srinivasan.
\end{abstract}

\section{INTRODUCTION}

Let $A$ be a real algebra (assumed to be nonzero and associative throughout). The celebrated Gelfand-Mazur Theorem [BD, Theorem 14.7, p. 73] states that if $A$ is a normed division algebra, then $A$ is isomorphic to the reals $\mathbb{R}$ or the complex numbers $\mathbb{C}$ or the quaternions $\mathbb{H}$. It follows from the recent work of Zalar [Z] that the same conclusion is obtained if $A$ is assumed to be a pre-Hilbert space satisfying either (i) $A$ has identity $1,\|1\|=1,\left\|a^{2}\right\| \leq\|a\|^{2}$ for all $a \in A$; or (ii) $\left\|a^{2}\right\|=\|a\|^{2}$ for all $a \in A$. Theorem 1 below is a " $C^{*}$-property analogue" of this "square property" result, thereby strengthening the analogy between (certain aspects of) $C^{*}$-algebras and uniform Banach algebras envisaged in [B]. Theorem 1 is also closely related with the Froelich-Ingelstam-Smiley Theorem concerning unital multiplication in Hilbert space [Fr].

Theorem 1. Let $\|\cdot\|$ be a Pythagorean norm on a real ${ }^{*}$-algebra $A$.

(a) Assume that $\|\cdot\|$ satisfies at least one of the following.

(i) (1) A has identity, $\|1\|=1,\left\|a^{*} a\right\| \leq\|a\|^{2}$ for all $a \in A$, and (2) $a^{*} a=0 \Rightarrow$ $a=0,(a \in A)$.

(ii) $\left\|a^{*} a\right\|=\|a\|^{2}$ for all $a \in A$.

Then $A$ is isomorphic to $\mathbb{R}$ or $\mathbb{C}$ or $\mathbb{H}$.

(b) Suppose A is a complex *-algebra. Assume that at least one of the following holds.

(i) $A$ has identity, $\left\|a^{*} a\right\| \leq\|a\|^{2}$ for all $a \in A$ and $\|1\|=1$.

(ii) $\left\|a^{*} a\right\|=\|a\|^{2}$ for all $a \in A$.

Then $A \cong \mathbb{C}$.

Received by the editors September 23, 1996 and, in revised form, March 12, 1997.

1991 Mathematics Subject Classification. Primary 46K15, 46H20.

Key words and phrases. Real *-algebra, Pythagorean norm, Gelfand-Mazur Theorem, FroelichIngelstam-Smiley Theorem.

(c) 1998 American Mathematical Society 
A Pythagorean norm on $A$ is a norm $\|\cdot\|$ on $A$ such that $(A,\|\cdot\|)$ is a normed linear space satisfying

$$
\|a+b\|^{2}+\|a-b\|^{2}=2\|a\|^{2}+2\|b\|^{2} \quad(a, b \in A) .
$$

We shall show that in (a) above, the condition (2) in (i) cannot be omitted. Thus a comparison of (a) and (b) brings out an essential difference between real *-algebras and complex *-algebras. Theorem 1 will be derived from the following more general result.

Theorem 2. Let $A$ be a real *-algebra with identity 1. Let $\operatorname{Sym} A=\left\{a \in A: a^{*}=\right.$ a\}. Assume that

$$
a^{*} a=0 \text { implies } a=0 \text { for } a \in A \text {. }
$$

Let $\|\cdot\|$ be a norm on $\operatorname{Sym} A$. Suppose for all $a, b$ in Sym $A$ satisfying $a b=b a$, that

$$
\|a+b\|^{2}+\|a-b\|^{2}=2\|a\|^{2}+2\|b\|^{2} .
$$

Assume that at least one of the following holds.

(i) $\|1\|=1,\left\|a^{2}\right\| \leq\|a\|^{2}(a \in \operatorname{Sym} A)$.

(ii) $\left\|a^{2}\right\|=\|a\|^{2}$ for all $a \in \operatorname{Sym} A$.

Then $A$ is isomorphic to $\mathbb{R}$ or $\mathbb{C}$ or $\mathbb{H}$.

A real $C^{*}$-algebra $[\mathrm{G}]$ is a real *-algebra $A$ with a norm $\|\cdot\|$ such that $\|a b\| \leq$ $\|a\|\|b\|,\left\|a^{*} a\right\|=\|a\|^{2}$ for all $a, b \in A$; and for each $a \in A, 1+a^{*} a$ is invertible in the unitization $A_{e}$ of $A$.

Theorem 3. (a) Let $A$ be a real $C^{*}$-algebra. Assume that at least one of the following holds.

(i) A has identity, $|h|= \pm h$ for all $h \in \operatorname{Sym} A$.

(ii) Sym $A$ has no nonzero zero divisor.

Then $A$ is isomorphic to $\mathbb{R}$ or $\mathbb{C}$ or $\mathbb{H}$.

(b) Let $A$ be a complex $C^{*}$-algebra satisfying at least one of the above (i) or (ii) or the following

(iii) $A$ is unital and for each $h \in \operatorname{Sym} A$, $e^{i h}$ has convex spectrum.

Then $A$ is isomorphic to $\mathbb{C}$.

Part (b) of the above theorem with assumption (iii) improves a known result that a complex unital $C^{*}$-algebra in which each unitary element has convex spectrum is isomorphic to $\mathbb{C}$. We do not know an appropriate real analogue of assumption (iii). Theorem 3 with assumption (ii) throws further light on the comparison between $C^{*}$-algebras and uniform Banach algebras mentioned above in view of the fact that there are nontrivial uniform algebras (e.g. the disc algebra) that are integral domains. Further, Kaplansky's version of the Gelfand-Mazur Theorem states that a real normed algebra admitting no nonzero topological zero divisors is isomorphic to $\mathbb{R}$ or $\mathbb{C}$ or $\mathbb{H}[\mathrm{GP}]$. Theorem 3 shows that in the presence of the $C^{*}$-property, the assumption concerning topological zero divisors can be weakened. The following exhibits another such instance.

Theorem 4. Let $A$ be a real Banach algebra with identity 1. Suppose that $A$ has no nonzero zero divisors and $A$ is locally finite. Then $A$ is isomorphic to $\mathbb{R}$ or $\mathbb{C}$ or $\mathbb{H}$. 
This improves a result of Srinivasan [S]. He proved it for commutative algebras over $\mathbb{C}$. However, the proof by Srinivasan contains a gap which was rectified by Heinze $[\mathrm{He}]$ who proved that an associative locally finite algebra which is an integral domain (hence commutative) over an algebraically closed field is isomorphic to the ground field.

A (complex or real) normed algebra $(A,\|\cdot\|)$ with identity 1 is locally finite if for each $h \in A$ the smallest (not necessarily closed) subalgebra containing $h$ and 1 is finite dimensional.

\section{ProOfs}

(2.1) Lemma. Let $A$ be a unital real ${ }^{*}$-algebra satisfying $a^{*} a=0$ implies $a=0$ $(a \in A)$.

(i) If Sym $A$ is isomorphic to $\mathbb{R}$, then $A$ is ${ }^{*}$-isomorphic to $\mathbb{R}$ or $\mathbb{C}$ or $\mathbb{H}$ with the usual involution.

(ii) If Sym $A$ is isomorphic to $\mathbb{C}$, then $A$ is ${ }^{*}$-isomorphic to $\mathbb{C}$ with the identity involution.

Proof. Define Skew $A=\left\{a \in A: a^{*}=-a\right\}$. Then $A=\operatorname{Sym} A \oplus \operatorname{Skew} A$. Let $a \in A, a \neq 0$. By the assumption, $a^{*} a \neq 0$. Since $a^{*} a \in \operatorname{Sym} A$, and since $\operatorname{Sym} A$ will be assumed to satisfy either the hypothesis of (i) or (ii), then $a^{*} a$ is a nonzero real or complex number, hence invertible. It follows now that $a$ is invertible in $A$. Thus $A$ is a division algebra. (Note that if $A$ is a normed algebra, this immediately gives the conclusion).

Case (i). Suppose $\operatorname{Sym} A \cong \mathbb{R}$.

Let $a \in A$. Then $a=h+k$ for some $h \in \operatorname{Sym} A, k \in$ Skew $A$. Thus $(a-h)^{2}=$ $k^{2}-k^{*} k$. Since $h$ and $k^{*} k$ are in $\operatorname{Sym} A$, we have $a=\alpha 1, k^{*} k=\beta 1$ for some $\alpha, \beta$ in $\mathbb{R}$. Then $(a-\alpha)^{2}+\beta=0$. Thus every element in $A$ is algebraic over $\mathbb{R}$ (in fact, of degree $\leq 2$ ). Hence by the Frobenius Theorem, $A$ is isomorphic to $\mathbb{R}, \mathbb{C}$ or $\mathbb{H}$.

Case (ii). Suppose Sym $A \cong \mathbb{C}$.

Let $u \in \operatorname{Skew} A$. Let $w=i u+u i$. Since $u^{*}=-u$ and $i^{*}=i$ as $i \in \operatorname{Sym} A$, we have $w^{*}=-u i-i u=-w \in$ Skew $A$. Further, $i w=-u+i u i, w i=i u i-u$; and so $w \lambda=\lambda w$ for all $\lambda=\lambda 1 \in \mathbb{C}$. Also $w^{2}=-w^{*} w \in \operatorname{Sym} A$; and so $w^{2}=\alpha^{2}$ for some $\alpha \in \mathbb{C}$. Now since $w \alpha=\alpha w$, we have $(w-\alpha)(w+\alpha)=w^{2}-\alpha^{2}=0$. Thus $w= \pm \alpha \in \mathbb{C}$, i.e., $w \in \operatorname{Sym} A$. Now $w=0$ as $w \in \operatorname{Sym} A \cap \operatorname{Skew} A$. Thus $i u+u i=0$. Hence $i u=-u i=(i u)^{*}, i u \in \operatorname{Sym} A \cong \mathbb{C}$, and so $u \in \mathbb{C}=\operatorname{Sym} A$. Thus $u \in \operatorname{Sym} A \cap \operatorname{Skew} A=\{0\}$. We have shown that Skew $A=\{0\}$. Hence $A=\operatorname{Sym} A \cong \mathbb{C}$.

(2.2) Lemma. Let $A$ be a real ${ }^{*}$-algebra with identity 1 . Let $\|\cdot\|$ be a norm on $\operatorname{Sym} A$. Suppose for all $a, b$ in $\operatorname{Sym} A$ with $a b=b a$, that $\|a+b\|^{2}+\|a-b\|^{2}=$ $2\|a\|^{2}+2\|b\|^{2}$. Assume that at least one of the following holds.

(i) $\|1\|=1,\left\|a^{2}\right\| \leq\|a\|^{2}$, for all $a \in \operatorname{Sym} A$.

(ii) $\left\|a^{2}\right\|=\|a\|^{2}$ for all $a \in \operatorname{Sym} A$.

Then $\operatorname{Sym} A$ is isomorphic to $\mathbb{R}$ or $\mathbb{C}$.

Proof. Let $a \in \operatorname{Sym} A$. Let $P$ denote the algebra of all polynomials $p(x)$ with real coefficients in one indeterminate $x$ including the constants. Let $B=\{p(a): p \in P\}$. Then $B$ is a commutative algebra contained in Sym $A$. Thus $B$ is a real commutative algebra with 1 satisfying $\|1\|=1$ and $\left\|u^{2}\right\| \leq\|u\|^{2}$ for all $u \in B$. Also, $B$ is an inner product space. Hence by $[\mathrm{Z}$, Theorem 1 , p. 1418], $B \cong \mathbb{R}$ or $\mathbb{C}$. (Of course, 
being commutative $B$ cannot be quaternions or octonions.) Hence $a=\lambda 1$ for some $\lambda \in \mathbb{R}$ or $\mathbb{C}$. Hence $\operatorname{Sym} A \cong \mathbb{R}$ or $\mathbb{C}$.

(2.3) Theorem 2 follows immediately from Lemmas 2.1 and 2.2 .

(2.4) Proof of Theorem 1. (a) (i) follows immediately from Theorem 2.

(ii) In view of (i) it is enough to show that $A$ has identity 1 and $\|1\|=1$. Let $a \in \operatorname{Sym} A$ be a nonzero element of $A$. Let $B$ be the algebra of all polynomials in $a$ with real coefficients without constant terms. Then $B$ is a commutative algebra with a Pythagorean norm. Further, since every $u \in B$ is selfadjoint, we have $\left\|u^{2}\right\|=\left\|u^{*} u\right\|=\|u\|^{2}$ for all $u \in B$. Hence by [Z, Theorem 3], $B$ is isomorphic to $\mathbb{R}$ or $\mathbb{C}$ (since $B$ is commutative it cannot be isomorphic to $\mathbb{H}$ ). In particular, $B$ has a unit, say $e$. We show below that $e$ is the identity of $A$ as well. Our argument is similar to the one in [Z, Theorem 3].

Note that $e^{2}=e,\|e\|=1$ and $e^{*}=e$. Let $x \in A$. Suppose that $b=x-x e \neq 0$. Now $b e=0$. Let $y=\frac{b^{*} b}{\|b\|^{2}}$. Then $y^{*}=y,\|y\|=1$ and $y e=0$. Consequently, $(y e)^{*}=e y=0$. Since $(e+y)^{*}=e^{*}+y^{*}=e+y$, we have $\|e+y\|^{2}=\left\|(e+y)^{2}\right\|=$ $\left\|e^{2}+e y+y e+y^{2}\right\|=\left\|e+y^{2}\right\|=\left\|e-e y-y e+y^{2}\right\|=\left\|(e-y)^{2}\right\|=\|e-y\|^{2}$. Hence, $\|e+y\|^{2}=(1 / 2)\left\{\|e+y\|^{2}+\|e+y\|^{2}\right\}=(1 / 2)\left\{\|e+y\|^{2}+\|e-y\|^{2}\right\}=$ $(1 / 2)\left\{2\|e\|^{2}+2\|y\|^{2}\right\}=2$. Then $4=\|e+y\|^{4}=\left\{\|e+y\|^{2}\right\}^{2}=\left\|e+y^{2}\right\|^{2}=$ $\left\langle e+y^{2}, e+y^{2}\right\rangle=\|e\|^{2}+\left\|y^{2}\right\|^{2}+2\left\langle e, y^{2}\right\rangle=2+2\left\langle e, y^{2}\right\rangle$. Thus $\left\langle e, y^{2}\right\rangle=1=\|e\|\left\|y^{2}\right\|$ (the equality in the Schwarz inequality). And so, $e=y^{2}$. But then $1=\|y\|^{4}=$ $\left\|y^{2}\right\|^{2}=\left\|y^{4}\right\|=\left\|y^{2} e\right\|=0$. This shows that $b=0$, i.e., $x e=x$. Similarly, $e x=x$. This completes the proof of (a).

(b) (i) Assume (i). So $A$ is a real *-algebra. So, by Lemma 2.2(i), Sym $A \cong \mathbb{R}$ or $\mathbb{C}$. Since $A$ is a complex algebra $i^{*}=-i$, as a result $\operatorname{Sym} A$ must be $\mathbb{R}$. But then $A \cong \operatorname{Sym} A \oplus i \operatorname{Sym} A=\mathbb{R} \oplus i \mathbb{R} \cong \mathbb{C}$.

(ii) Assume (ii). By (a) (ii) $A \cong \mathbb{R}$ of $\mathbb{C}$ or $\mathbb{H}$. Being a complex algebra $A \cong \mathbb{C}$. (Alternatively, the completion $B$ of $A$ is a $C^{*}$-algebra which is a complex Hilbert space, hence $B$ is strictly convex. Therefore, $\{x \in B:\|x\|=1\}$ is the set of all extreme points of $\{x \in B:\|x\| \leq 1\}$. As a result, $B$ has an identity 1 and $\|1\|=1$.)

(2.5) Proof of Theorem 3. (a) Let $a \in \operatorname{Sym} A$ and $C$ be the $\|\cdot\|$-completion of the subalgebra $B$ of $A$ generated by $a$ and 1 . Note that $C$ can be identified with a subalgebra of $A$. Hence, if $A$ satisfies (i) or (ii), then so does $C$. Therefore $C$ is a commutative real $C^{*}$-algebra $[\mathrm{G}]$ in which the spectrum of every element is contained in the real line. Hence by an ancient Theorem of Arens, $C$ is isomorphic to $C_{\mathbb{R}}(X)$ for some compact Hausdorff space $X$. Let $x_{1}, x_{2}$ be two distinct points of $X$. If (i) holds, then by Urysohn's lemma, there exists $h \in C_{\mathbb{R}}(X)$ such that $h\left(x_{1}\right)=1, h\left(x_{2}\right)=-1$. But then $|h| \neq \pm h$, contradicting (i). If (ii) holds, then by a similar argument there exist $g_{1}, g_{2}$ in $C_{\mathbb{R}}(X)$ such that $g_{1} \neq 0 \neq g_{2}$ but $g_{1} g_{2}=0$, contradicting (ii). Thus, in any case $X$ must be a singleton and hence $a$ must be a real multiple of the identity. So $\operatorname{Sym} A \cong \mathbb{R}$. This proves (a) by Lemma 2.1.

(b) If (i) or (ii) holds, then arguments parallel to that in (a) prove the conclusion. So assume (iii). Let us fix $h \in \operatorname{Sym} A$. Since $e^{i h}$ is a unitary element, $\operatorname{sp}\left(e^{i h}\right) \subset$ $\Gamma=\{z \in \mathbb{C}:|z|=1\}$. But the only convex subsets of $\Gamma$ are singletons. So, $\operatorname{sp}\left(e^{i h}\right)$ must be a singleton. Now let $\lambda_{1}, \lambda_{2} \in \operatorname{sp}(h)$ and $\lambda_{1}<\lambda_{2}$. Define

$$
y=\frac{2\left(h-\lambda_{1}\right)}{\lambda_{2}-\lambda_{1}}+1
$$


It is clear that $y \in \operatorname{Sym} A$. Since $\lambda_{1}, \lambda_{2} \in \operatorname{sp}(h), 1,2 \in \operatorname{sp}(y)$. As a result $e^{i}, e^{2 i} \in$ $\operatorname{sp}\left(e^{i y}\right)$, which is a contradiction. As a result, $\operatorname{sp}(h)$ must be a singleton and the proof follows.

The following is a real analogue of [S, Theorem 2.2].

(2.6) Lemma. Let $A$ be a real Banach algebra which is an integral domain. The following conditions are equivalent for $h \in A$.

(i) $h$ is not a topological divisor of zero.

(ii) The principal ideals $A h$ and $h A$ generated by $h$ are closed.

Proof. Suppose $A h$ is not closed. Then there is a sequence $\left\{a_{n} h\right\}$ in $A$ such that $a_{n} h \rightarrow a$ and $a \notin A h$. It follows that $\left\{a_{n}\right\}$ is not a Cauchy sequence (otherwise $a_{n} \rightarrow b$ implies $\left.a=b h \in A h\right)$. Since $\left\{a_{n}\right\}$ is not Cauchy there exists an $\varepsilon>0$ and subsequence $\left\{a_{n_{i}}\right\}$ of $\left\{a_{n}\right\}$ such that $\left\|a_{n_{i}}-a_{n_{j}}\right\|>\varepsilon$ for all $i, j$. Let $b_{n_{i}}=$ $\frac{a_{n_{i}}-a_{n_{i+1}}}{\left\|a_{n_{i}}-a_{n_{i+1}}\right\|}$. Then $\left\|b_{n_{i}}\right\|=1$ and $b_{n_{i}} h \rightarrow 0$. Thus $h$ is a topological divisor of zero. Similarly if $h A$ is not closed, then $h$ is also a topological divisor of zero.

Conversely, suppose that $A h$ and $h A$ are closed. Define $R_{h}: A \rightarrow(A h)$ by $R_{h}(a)=a h(a \in A)$ and $L_{h}: A \rightarrow(h A)$ by $L_{h}(a)=h a(a \in A)$. Since $A$ is an integral domain, $L_{h}$ and $R_{h}$ are one-one. But then since $h A$ and $A h$ are closed, the open mapping theorem implies that they are homeomorphisms. So there exist $M_{1}, M_{2}, N_{1}, N_{2}>0$ such that $M_{1}\|a\| \leq\|a h\| \leq M_{2}\|a\|$ and $N_{1}\|a\| \leq\|h a\| \leq$ $N_{2}\|a\|$. Now for any sequence $\left\{a_{n}\right\}$ in $A, a_{n} h \rightarrow 0$ or $h a_{n} \rightarrow 0$ implies that $a_{n} \rightarrow 0$. Hence $h$ cannot be a topological divisor of zero.

(2.7) Corollary. Let a real Banach algebra $A$ be an integral domain in which all principal ideals are closed. Then $A$ is isomorphic to $\mathbb{R}$ or $\mathbb{C}$ or $\mathbb{H}$.

Proof. By the above theorem $A$ does not contain a topological divisor of zero. Hence by the real version of Kaplansky's result, $A$ is isomorphic to $\mathbb{R}$ or $\mathbb{C}$ or $\mathbb{H}$.

(2.8) Proof of Theorem 4. Let $a \in A$ and $C$ be the $\|\cdot\|$-completion of the algebra $B$ generated by $a$ and 1 . Then $C$ is a commutative unital finite dimensional algebra with 1 which is an integral domain. Because of the finite dimensionality of $C$ all the ideals (and hence all the principal ideals) of $C$ are closed. Consequently, $C$ does not contain any topological divisors of zero. But then $C$ is isomorphic to $\mathbb{R}$ or $\mathbb{C}$ or $\mathbb{H}$. As a result, $a$ is invertible in $C$ and hence in $A$. Now the conclusion follows by the classical Gelfand-Mazur Theorem.

\section{REMARKS}

(3.1) Let $u \neq 0$ be such that $u^{2}=0$, e.g. $u=\left(\begin{array}{ll}0 & 1 \\ 0 & 0\end{array}\right)$. Let $A=\{\alpha+\beta u: \alpha, \beta \in \mathbb{R}\}$, $(\alpha+\beta u)^{*}=\alpha-\beta u$. Then $A$ is a unital real *-algebra in which $\operatorname{Sym} A \cong \mathbb{R}$. However, $A$ is not isomorphic to $\mathbb{R}$ or $\mathbb{C}$ or $\mathbb{H}$. This shows that the condition $a^{*} a=0 \Rightarrow a=0$ cannot be omitted from Lemma 2.1 as well as from Theorem 1(a)(i).

(3.2) The algebra $\mathbb{R} \times \mathbb{R}$ with pointwise multiplication and the Euclidean norm $\left\|\left(z_{1}, z_{2}\right)\right\|_{2}=\left(\left|z_{1}\right|^{2}+\left|z_{2}\right|^{2}\right)^{1 / 2}$ satisfies everything in Theorem 1(a)(i) except $\|1\|_{2}=$ 1. Thus we cannot drop the condition of the norm being unital in (i) of (a) and (b) of Theorem 1. This also shows that the condition $\left\|a^{*} a\right\| \leq\|a\|^{2}$ cannot be weakened to $\left\|a^{*} a\right\| \leq K\|a\|^{2}(a \in A)$. Indeed on $\mathbb{R}^{2},\left|\left(z_{1}, z_{2}\right)\right|=(1 / 2)^{1 / 2}\left\|\left(z_{1}, z_{2}\right)\right\|_{2}$ defines 
a Pythagorean norm having $|1|=1,\left|a^{*} a\right| \leq 2^{1 / 2}|a|^{2}\left(a \in \mathbb{R}^{2}\right)$. Also, the nonPythagorean max-norm $\|\cdot\|_{\infty}$ on $\mathbb{R}^{2}$ is equivalent to $\|\cdot\|_{2}$ and satisfies $\|1\|_{\infty}=1$. Thus the condition that $\|\cdot\|$ is Pythagorean cannot be replaced by the condition that $\|\cdot\|$ is equivalent to a Pythagorean norm.

(3.3) The disc algebra $A(\mathbb{D})$ with the involution $f^{*}(z)=\overline{f(\bar{z})}$ and norm $|f|=$ $\sup \{|f(z)|: z \in[-1,1]\}$ illustrates that completeness cannot be dropped from Theorem 3 (b) (ii).

(3.4) (i) Let $A$ be a hermitian Banach *-algebra. Assume (iii) of Theorem 3. Our proof shows that $A$ is $\mathbb{R}$ or $\mathbb{C}$ or $\mathbb{H}$. Does this hold if instead we assume that $A$ has no nonzero zero divisors?

(ii) Let $A$ be a *-semisimple Banach *-algebra with unique (not necessarily complete) $C^{*}$-norm. Let $A$ have no nonzero zero divisors. Is $A$ isomorphic to $\mathbb{R}$ or $\mathbb{C}$ or $\mathbb{H}$ ?

(iii) Let $A$ be a unital linear associative algebra. Let $p$ be a seminorm on $A$ such that $p(1)=1$. Suppose either (a) $p\left(a^{2}\right) \leq p(a)^{2}$ for all $a$; or (b) $A$ is a ${ }^{*}$-algebra and $p\left(a^{*} a\right) \leq p(a)^{2}$ for all $a$. If $p$ is Pythagorean, does it follow that $p(a b) \leq p(a) p(b)$ for all $a, b$ in $A$ ? If $A$ is commutative and if (a) holds, then this is true. (Indeed given $a, b$ in $A, a b=b a$ gives $4 a b=(a+b)^{2}+(a-b)^{2}$. Hence

$$
\begin{aligned}
4 p(a b) & \leq p\left((a+b)^{2}\right)+p\left((a-b)^{2}\right) \\
& \leq(p(a+b))^{2}+(p(a-b))^{2}=2 p(a)^{2}+2 p(b)^{2} .
\end{aligned}
$$

Hence $p(a) \leq 1, p(b) \leq 1$ implies $p(a b) \leq 1$. It follows that $p(a b) \leq p(a) p(b)$ for all $a, b$.) In the absence of the Pythagorean assumption the power inequality $p\left(a^{n}\right) \leq p\left(a^{n}\right)(a \in A, n \in \mathbb{N})$ does not imply $p(a b) \leq p(a) p(b)$, as is exhibited by the numerical radius in Banach algebras. Does weak submultiplicativity $p(a b) \leq$ $M p(a) p(b)$ follow under any of the assumptions (a) or (b)?

(iv) A comparison of Theorem 1 with the $C^{*}$-algebra characterization by GlimmKadison as well as by Vowden [BD, Theorem 15, p. 212] suggests: Can we replace $\left\|a^{*} a\right\| \leq\|a\|^{2}$ (respectively, $\left\|a^{*} a\right\|=\|a\|^{2}$ ) by $\left\|a^{*} a\right\| \leq\left\|a^{*}\right\|\|a\|$ (respectively, by $\left.\left\|a^{*} a\right\|=\left\|a^{*}\right\|\|a\|\right)$ in Theorem 1 ?

\section{ACKNOWLEDGEMENTS}

The authors are thankful to the referee for a very careful reading of the paper.

\section{NOTE ADDED IN PROOF}

In connection with the question discussed in remark 3.4(iii), lately it has come to our notice that R. Arens, M. Goldberg and W. A. J. Luxemburg (Multiplicativity factors for seminorms II, J. Math. Anal. Appl. 170(2) (1992), 401-413) have shown that (a) if $p$ is a seminorm on a commutative algebra $A$ satisfying (iii)(a), then $p(x y) \leq 2 p(x) p(y)(x, y \in A)$ where the inequality is best possible, and (b) there exist non-commutative algebras with non-weakly submultiplicative seminorms and norms satisfying (iii)(a) above. 


\section{REFERENCES}

[B] S. J. Bhatt, Some remarks on hermitian Banach * -algebras, Indian J. Pure Appl. Math. 26 (2) (1995), 131-142. MR 96e:46065

[BD] F. F. Bonsall, and J. Duncan, Complete Normed Algebras, Springer-Verlag, Berlin, Heidelberg, New York, 1973. MR 54:11013

[Fr] J. Froelich, Unital multiplication on a Hilbert space, Proc. Amer. Math. Soc. 117 (1993), 757-759. MR 93d:46081

[G] K. R. Goodearl, Notes on Real and Complex $C^{*}$-Algebras, Shiv Publ. Co., England, 1987. MR 85d: 46079

[GP] M. C. Garcia and A. R. Palacios, A new simple proof of the Gelfand-Mazur Theorem, Proc. Amer. Math. Soc. 123 (9) (1995), 2663-2666. MR 95k:46077

[He] J. Heinze, A remark on a paper of V. K. Srinivasan, Bull. Austral. Math. Soc. 22 (1980), 153-154. MR 82a:46045

[S] V. K. Srinivasan, On Some Gelfand-Mazur like theorems in Banach algebras, Bull. Austral. Math. Soc. 20 (1979), 211-215; Corrigenda, ibid 23 (1981), 497-480. MR 81e:46029a; MR 82h: 46067

[Z] B. Zalar, On Hilbert space with unital multiplication, Proc. Amer. Math. Soc. 123 (1995), 1497-1501. MR 95f:46093

(S. J. Bhatt and D. J. Karia) Department of Mathematics, Sardar Patel University, Vallabh Vidyanagar-388 120, Gujarat, India

(S. H. Kulkarni) Department of Mathematics, Indian Institute of Technology, Madras, 600 036, INDIA

(M. E. Shimpi) Department of Mathematics, BVM Engineering College, Vallabh Vidyanagar-388 120, Gujarat, India 